\title{
Research on Driving Behavior Simulation Based on Adaptive Agent
}

\author{
Qianjiao $\mathrm{Wu}^{1}$, Rong $\mathrm{Lan}^{2}$ and Wei Zhou ${ }^{3}$ \\ ${ }^{1}$ School of information engineering, Chang 'An University, Xi 'An, 710064, PR China \\ ${ }^{2}$ School of economics and finance, Xi 'an Jiao Tong University, Xi 'An, 710061, PR China \\ ${ }^{3}$ China Academy of Transportation Sciences Beijing, 100088, PR China
}

\begin{abstract}
Papers use adaptive thinking ${ }^{[4]}$ and Boid model and Couzin et al. ${ }^{[6][7][8]}$ mathematical description Boid model method, described the vehicle as the driver based on the current state of environment and adapt to the individual behavior, the driver's driving process is a process of adapted to the environment of the vehicle. Paper to establish the decision-making behavior of single vehicle adaptive scene adaptability of driving behavior description, to expect goals in the process of driver's driving as decisionmaking basis, defines the three driving state, and analyzes the driving behavior and decision rules. Simulation results indicate: The behavior changes of the individual adaptive driving vehicles are completely consistent with the characteristics of the macrotraffic flow, showing the cooperative characteristics of the group agent; Individual driving vehicles show good adaptability and selfdrive to the change of environment and current traffic condition.; The change of individual driving behavior of the vehicle will have an impact on the adjacent local vehicle individuals; The change of traffic density affects the speed of traffic flow; Bad driving behavior will affect the local and even the whole road traffic speed, can cause the vehicle queue together and split the queue phenomena, things will appear serious traffic queue phenomenon, resulting in a decline in the capacity of the road.
\end{abstract}

Keywords-traffic simulation; driving behavior; Boid model; adaptive agent

\section{INTRODUCTION}

Reuschel. A. (1950) ${ }^{[1]}$ and Pipes L.A. (1953) $)^{[2]}$ using the dynamics of the vehicle before and after the analysis of motion process to get to know one lane traffic flow characteristics, microscopic driver behavior and macroscopic traffic flow phenomena, form the car following theory.

Literature [3] systematically reviewed the development process current situation of car following theory, classified the model, and expounded various model ideas, structures and parameters. After decades of development, the car following theory has become one of the core contents of traffic flow theory, and it is the theoretical basis of traffic flow simulation, road capacity and intelligent driving.

In the process of development, because of the driver's psychological and physiological, intelligence and decisionmaking for the pilot culture, individual differences factors such as age, gender, regional, unable to accurately evaluate all kinds of models.

Considering that driving behavior is the embodiment of human-vehicle-road-environment interaction, the driver is always the decision maker and operator of driving behavior, and driving behavior cannot be simply regarded as a purely mechanical process.

Therefore, the study of driving behavior is an important direction. The research on driving behavior is helpful to the development of intelligent auxiliary driving and unmanned driving.

Drivers drive double restriction by travel efficiency and safety, in order to achieve the desired efficiency of travel, the driver is on the basis of traffic conditions at that time as far as possible to the desired speed, show the following leading into, at the same time to avoid collision with leading vehicle

In the course of driving, the driver continuously makes the perception decision and realizes the control on the change of the driving environment and traffic state, and the vehicle driving state always changes with the current traffic flow state

The change of driving environment is related to the objective conditions of current road driving, such as the density of traffic flow and average speed of current road.

Therefore, when driving, the driver always seeks a suitable workshop distance to ensure the time to complete the relevant operation and meet the psychological expectation of the driver. This psychological expectation of driving is constantly changing with the change of driving environment. See figure 1.

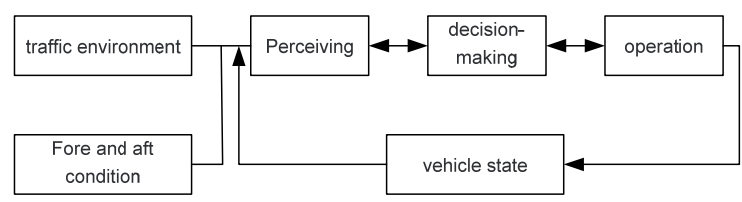

FIGURE I. DECISION-MAKING PROCESS OF DRIVER'S ROAD DRIVING

Literature [4] defines from a biological perspective the process by which an organism adjusts itself to the environment as adaptation. In the process of adaptation, situations and activities are constantly changing. The utility of the various activities of a particular organism often depends to a large extent on the ever-changing scenarios provided by other organisms. This's a complex adaptive system (CAS). Although different CAS processes have different time scales, the concept can be applied to all CAS subjects. The CAS diversity is a dynamic model with persistence and coordination. It is the result of constant adaptation. Every new adaptation lays the foundation for further interaction. 
Similarly, the driving process of the driver is a process of adapting to the environment of the driving vehicle. That is, a decision and judgment between the current traffic environment, the permitted driving condition and the driving condition required by the driving vehicle. Therefore, the driver's driving vehicle is set as an agent. The driving behavior of a vehicle is described as the response of an intelligent body to circumstances and conditions. Traffic flow is regarded as the result of the coordination or cooperation between groups of agent.

\section{DEscription OF AdAPTIVE Driving BEHAVIOR}

In this paper, using the ideas of intelligent body and CAS, reference Reynolds Boid mode ${ }^{[5]}$, with the help of a Couzin et al. ${ }^{[6][7][8]}$ mathematical description Boid model method and the movement rules, described the vehicle as the driver based on the current state of environment and adapt to the individual behavior, so the driver's driving process is a process and the environmental adaptation of the vehicle.

The driver's vehicle behavior is described as the response of an agent body to the environment and conditions. According to the idea of Boid model, a simulation scenario of vehicle driving is constructed with a single lane road vehicle as an example. See figure 2 .

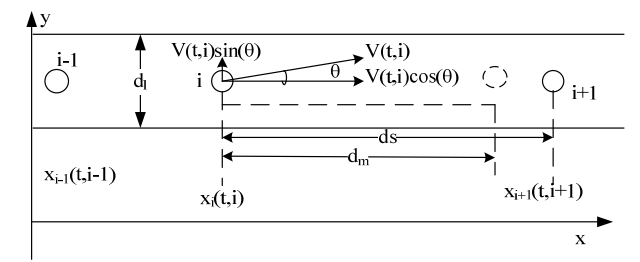

FIGURE II. STATE OF VEHICLE ADAPTIVE DECISION

Assumes that $n$ a running vehicle individual, each individual vehicle can movement in the whole $2 \mathrm{~d}$ space, but each individual can perceive a limited area. In the twodimensional space, the position and velocity vectors of individual $i(i=1,2, \cdots, n)$ of each driving vehicle are $x_{i}$ and $v_{i}$ respectively.

Drivers drive first to meet the requirements of safety, always need a safe space travel distance, when driving distance have a security threat, generally use the behavior to meet the safety conditions, such as slow when with sufficient space distance, the driver's behavior more freedom, can accelerate or decelerate or maintain the current situation. In the figure, the one-way road width is $d_{l}(m)$, vehicle $i$ motion between vehicle $i-1$ and $i+1$.

For time $t$, vehicleiposition $x_{i}(t, i)$,the speed is $v_{i}(t, i)$,And there is an Angle in the direction of the longitudinal direction (x axis).

Definition $1, d_{s}(t, i)$ is set as the distance between vehicle $i$ and the vehicle in front, and $d_{m}(t, i)$ is defined as the motion space required by vehicle $i$ at the current speed $v_{i}(t, i) \cos \theta$.

Definition 2. If $d_{s}(t, i)-d_{m}(t, i) \gg d_{m}(t, i)>0$, defined as the freedom of the vehicle to drive.
In a free state, the current vehicle movement space $d_{m}(t, i)$ and the driver's reaction time $t_{\tau}$ and the current vehicle speed $v(t, i)$, maximum speed for the road traffic laws associated conditions of the vehicles and drivers psychological expectations.

Definition 3. $\left|d_{s}(t, i)-d_{m}(t, i)\right| \leq \varepsilon$, defined as a dynamic equilibrium state.

Where, $\varepsilon$ is the boundary area of driver decision-making, where the driver has no intention of decision-making behavior and basically maintains the current driving state.

Definition4. $\varepsilon<\left|d_{s}(t, i)-d_{m}(t, i)\right| \leq \delta$,defined as a constraint or non-free state. In non free conditions, traffic conditions is restrained, the driver on the basis of the current space of the car and the current speed of the information such as plus/minus speed adjustment, to adapt to the current driving environment. Under the current speed, the adjustment of the driver space size of the $\delta=\left|d_{s}(t, i)-d_{m}(t, i)\right|$, and if $d_{s}(t, i)-d_{m}(t, i)<0$, cannot satisfy the safety conditions, drivers to slow down, on the contrary, where $d_{s}(t, i)-$ $d_{m}(t, i) \geq 0$, drivers could keep existing status, or acceleration.

\section{DRIVING DECISION BEHAVIOR RULES}

Known at time $t$, individual vehicle speed is $v_{i}(t, i)$, there is a physical drive space distance $d_{s}(t, i)$ and a driving demand for dynamic security distance, $d_{m}(t, i)$, the driver on the basis of $d_{s}(t, i)$ and $d_{m}(t, i)$ determine an expectation distance, if he can't meet the requirements of expectation distance, drivers to slow down the operation, similar Boid rejection of established Three - Circle model region (zone of repulsion), if exceed expectations of the distance, the driver to accelerate operation, similar to attract area (zone of Attraction), if the status is kept constant, it is similar to the zone of orientation of the three-circle model.

The algorithm process is as follows:

If

$$
\left|d_{s}(t, i)-d_{m}(t, i)\right| \leq \varepsilon
$$

Then

$$
v_{i}(t+\Delta t, i)=v_{i}(t+, i)
$$

End

If

$$
d_{s}(t, i)-d_{m}(t, i) \gg d_{m}(t, i)>0
$$

Then

$$
v_{i}(t+\Delta t, i)=v_{i}(t+, i)+\Delta v \times \Delta t
$$

End

If

$$
\varepsilon<\left|d_{s}(t, i)-d_{m}(t, i)\right| \leq \delta
$$


Then

$$
v_{i}(t+\Delta t, i)=v_{i}(t+, i) \pm \Delta v \times \Delta t
$$

End

\section{ANALYSIS OF DRIVING BeHAVIOR Process}

According to the change of environment and state, the driver makes decisions and conducts operations under the restriction of safe driving and travel efficiency. In the case of single lane, the driver's operation mainly includes accelerating and decelerating and maintaining the current speed. These behavior depends on the driver's state of mind, body state, the traffic situation, the current state of the local environment, traffic information (such as the average speed of the vehicle traffic density) and other related factors, the different drivers show different driving behavior.

If the driver feels safety threat during driving, the behavior of the driver is highly consistent. The driver will immediately slow down or speed up the vehicle to eliminate the safety hazards. The magnitude change of deceleration or acceleration depends on the intensity of the driver's perception of danger

Defining a danger coefficient $c_{s}$ to describe the extent to which this response is carried out by the driver.

$$
c_{S}=e^{-d_{x}(t, i)}
$$

The danger coefficient is obviously related to the safe driving space that the driver feels. The smaller the space, the greater the coefficient.

If the driver didn't feel safe in the process of driving, vehicle speed adjustment is decided by the status of the current drive space, and the driver showed a lot of expectations of travel speed target difference, its decision-making behavior related to the expected target, if the desired objective strong, responsive, speed adjusting the size of the corresponding increase, on the other hand, the response lag, speed adjustment range is small. In general, expected target size is associated with driving current adjust the size.

For driving behavior, the driver needs a reaction time $\left(t_{\tau}\right)$ from perception to decision execution and takes a continuous execution time $\left(t_{b}\right)$ for the driver to achieve the desired goal.

In the actual driving process, the driver will make judgment and decision according to the driving environment and current state, which will not be passively executed.

If the driver knows the status and traffic environment information of the current local neighboring vehicles and makes a judgment in advance, the reaction time can be eliminated or reduced, and the execution process can be completed in advance.

Therefore, the response time of the driver is related to the amount of information that the driver perceives the environment at this time.

Define a reaction coefficient $c_{\tau}$, the expression is as follows.

$$
c_{\tau}=e^{-r(t, i)}
$$

Where $r(t, i)$ represents the size of the horizon range.

Under the condition that the driver feels safe driving danger, the space distance $\left(d_{u}\right)$ required by the driver is shown as a braking process, and the expression is as follows.

$$
d_{u}=v_{i}(t, i) * t_{\tau} * c_{\tau}+\frac{k\left[\left(v_{i}\left(t+t_{b}, i\right) c_{S}\right)^{2}-v_{i}(t, i)^{2}\right]}{\emptyset g}
$$

Where, $t_{\tau}$ is driver reaction time, $v_{i}(t, i)$ is the current speed. $t_{b}$ Is the driver from the decision of braking deceleration to the completion of the braking process execution time, $g$ is the gravitational acceleration $\left(9.8 \mathrm{~ms}^{-2}\right) ; \varnothing$ Is the maximum adhesion coefficient of wheel and road surface, The value is generally around $0.8, k$ Is the equivalent inertia coefficient of vehicle no-load mass and rotating parts, when normal braking , $\mathrm{k}=1.09$.

The driver did not feel the threat of safety in the course of driving, and the expectation required by the driver to adjust the space distance $d_{f}$ was expressed in the following formula.

$$
d_{f}=\frac{1}{2} \times a \times t_{b}^{2}+v_{i}(t, i) \times t_{b}
$$

Where, $a$ is the speed adjustment range, which is the acceleration/deceleration value, $t_{b}$ is the execution time of the driver's operation.

In most cases, the deceleration process is often used in the case of security threats, and the acceleration process often occurs in the case of no security risks.

Combine (3) and (4) to define a dynamic space $d_{m}(t, i)$

$$
d_{m}(t, i)=f\left(v_{i}(t, i)=\sum_{k=1}^{2} \alpha_{k} \cdot v_{i}^{k}(t, i) \approx \beta \cdot e^{\gamma v_{i}(t, i)}\right.
$$

\section{SIMULATION ANALYSIS}

$\beta$ and $\gamma$ values in equation (5) are obtained by exponential fitting method using the vehicle video data, the result of the test analysis is $\beta=20.6, \gamma=0.195$.

The simulation road length is set as a closed interval of $260 \mathrm{~m}$, and the maximum vehicle speed is set as $120 \mathrm{~km} / \mathrm{s}$, The maximum acceleration is $8 \mathrm{~ms}^{-2}$, The number of vehicles is respectively8and 4,the vehicle is uniformly distributed at the beginning, initial velocity is 0 .

Figure 3 shows the number of vehicles 4 . The space-time diagram of vehicle operation without interference noise. The Xaxis represents the position of the vehicle $(\mathrm{m})$, the Y-axis represents time(s), and the following graphs are the same.

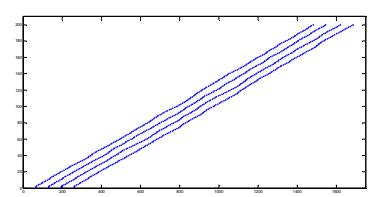

FIGURE III. VEHICLE NUMBER 4, SPACE-TIME DIAGRAM OF VEHICLE OPERATION WITHOUT INTERFERENCE NOISE 
Figure 4 shows the number of vehicles 8 . The space-time diagram of vehicle operation without interference noise.

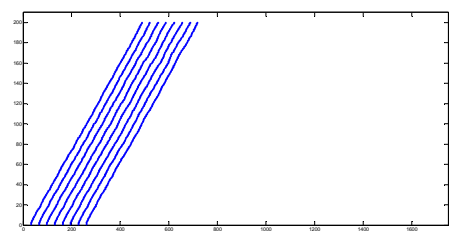

FIGURE IV. SHOWS THE NUMBER OF VEHICLES 8. THE SPACETIME DIAGRAM OF VEHICLE OPERATION WITHOUT INTERFERENCE NOISE

The comparison between FIGURE 3 and FIGURE 4 shows that the traffic density increases, the workshop spacing decreases, and the traffic flow speed decreases.

FIGURE 5(a), FIGURE 5(b) and FIGURE 5 (c) are the simulated space-time diagram when the number of vehicles is 4 , adding $1 \mathrm{db}$ noise.

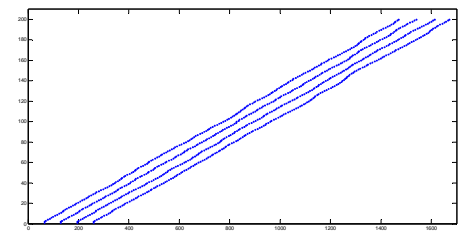

(a)

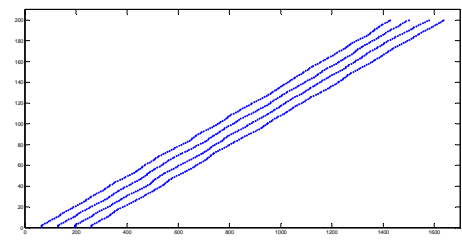

(b)

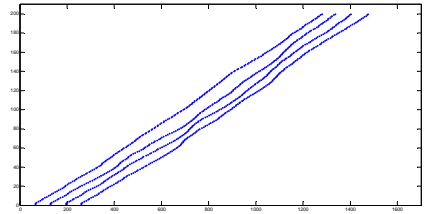

(c)

FIGURE V. VEHICLE NUMBER 4, THE SPACE-TIME DIAGRAM OF VEHICLE OPERATION ADDING 1db NOISE

Contrast figure 5(a)(b)(c) found that under the condition of same number of vehicles, the noise increased, the space between appear obvious uneven phenomenon, at the same time, the increase of traffic speed fluctuation, results in the decrease of the average speed of traffic.

Figure 6(a)(b)(c)(d) are simulated space-time diagrams of increasing $3 \mathrm{db}$ noise and vehicle number 8 .

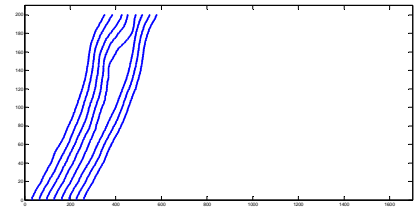

FIGURE VI. (a) VEHICLE NUMBER 8, ONE OF THE SIMULATION SPACE-TIME DIAGRAM OF INCRESING 3db NOISE

Figure 6(a) shows that noise can cause the phenomenon of vehicle alignment and separation. After adaptive intelligent decision making, the vehicle flow can be redistributed into a relatively uniform queue.

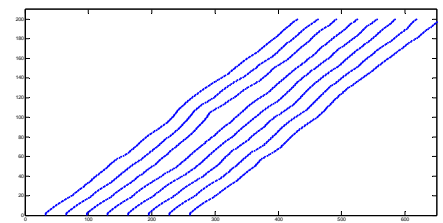

FIGURE VI. (b) VEHICLE NUMBER 8, ONE OF THE SIMULATION SPACE-TIME DIAGRAM OF INCRESING 3db NOISE

Figure 6(b) the local disturbance does not affect the overall condition of the vehicle flow queue.

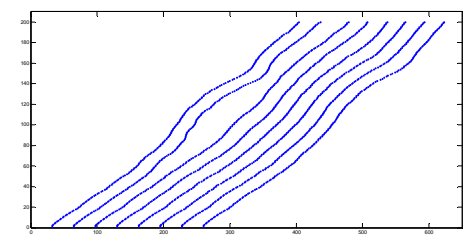

FIGURE VI. (c) VEHICLE NUMBER 8, ONE OF THE SIMULATION SPACE-TIME DIAGRAM OF INCRESING 3db NOISE

Figure6 (c) the local disturbance causes the fluctuation of the flow velocity of the entire flow queue and the decrease of the speed.

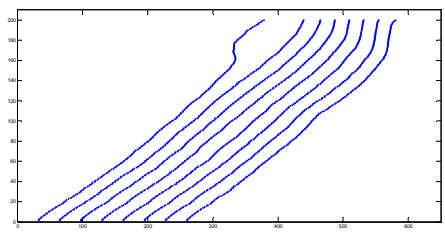

FIGURE VI. (d) VEHICLE NUMBER 8, ONE OF THE SIMULATION SPACE-TIME DIAGRAM OF INCRESING 3db NOISE

Figure 6(d) the parking or reversing phenomenon of individual vehicles will lead to a rapid decrease in the overall flow speed and a stop-and-go phenomenon.

Figure 7 is the local map of the two simulations under the same conditions. 

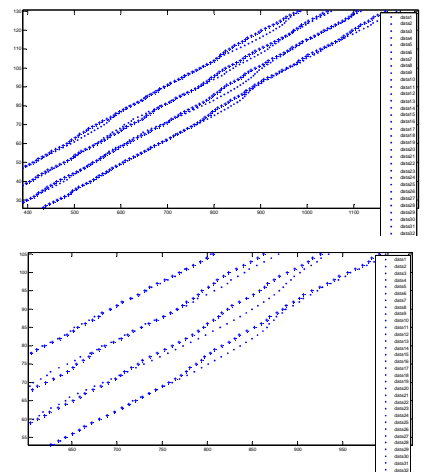

FIGURE VII. THE SPACE-TIME COMPARISON OF TWO REPEATED SIMULATION UNDER THE SAME CONDITIONS

\section{The Simulation Results}

In the initial state, the vehicle is set as a uniformly distributed area with zero speed, and the individual vehicle has the ability to drive itself.

Conclusion 1: individual vehicle to the change of environment and the current state of the traffic showed good adaptability

conclusion 2: the change of individual vehicles driving influence on adjacent subsequent local individual vehicles, define a speed change during the process of fetching a random disturbance noise with zero mean, short time disturbance affect local neighborhood of individual vehicles, not to expand, as the disturbance, affects the speed of traffic flow and traffic capacity. If the disturbance as driver driving behavior of the individual, bad driving behavior affects the traffic capacity of roads. The careful driving of drivers can effectively improve the influence of individual vehicle disturbance on the speed of the whole traffic flow. Increasing density of road traffic flow, the speed of the vehicle (including average speed).

\section{REFERENCES}

[1] REUSCHEL A. Vehicle Movements in the Column Uniformly Accelerated or Delayed[J]. Oesterrich Ingr Arch,1950.4,193-215.

[2] PIPES L A. An Operational Analyzation of Traffic Dynamics[J].Journal of Applied Physics ,1953,24(3):274-281.

[3] WANG Dian-hai , JIN Sheng. Review and Outlook Modeling of Car Following Behavior. China Journal Highway Transport,2012,115-127.

[4] John H. Holland. (Hidden Order: How Adaptation Builds Complexity. Perseus Books, 1996

[5] Reynolds C W. Flocks, herds and schools: A distributed behavioral model. ACM SIGGRAPH Computer Graph, 1987, 21: 25-34

[6] Couzin I D, Krause J, James R, et al. Collective memory and spatial sorting in animal groups. Theor Biol, 2002, 218: 1-11

[7] Couzin I D, Krause J, Franks N R, et al. Effective leadership and decisionmaking in animal groups on the move. Nature, 2005, 433: 513-516

[8] Couzin I D, Ioannou C C, Demirel G, et al. Uninformed individuals promote democratic consensus in animal groups. Science, 2011, 334: 